\title{
Profiles of Cytotoxic T Lymphocytes in Cutaneous Lymphoid Hyperplasia of the Face
}

\author{
Sadanori Furudate Taku Fujimura Yumi Kambayashi Setsuya Aiba \\ Department of Dermatology, Tohoku University Graduate School of Medicine, \\ Sendai, Japan
}

\section{Key Words}

Cutaneous lymphoid hyperplasia - Cutaneous diffuse large B cell lymphoma, not otherwise specified · Granulysin · Tumor-infiltrating lymphocytes · Cytotoxic T cells

\begin{abstract}
Cutaneous lymphoid hyperplasia (CLH) is difficult to differentiate from primary malignant cutaneous lymphomas that may present as solitary nodules, and sometimes it requires much time to achieve a final diagnosis. A recent report [Park et al.: Acta Haematol 2011;126:79-86] suggested that the expression of granulysin correlates with the prognosis of cancer patients, even in hematological disorders. In this report, we immunohistochemically examine the expression of cytotoxic molecules (e.g. granulysin, TIA-1 and perforin) in tumor-infiltrating lymphocytes of 10 patients with CLH and 3 patients with cutaneous diffuse large $B$ cell lymphoma, not otherwise specified (CDLBCL-NOS) of the face. In the patients with $\mathrm{CLH}$, the number of granulysin-bearing cells was higher than in the patients with CDLBCL-NOS. In contrast, there was no difference in the number of TIA $-1^{+}$or perforin ${ }^{+}$cells. The present study attempts to explain the different biological behaviors of these two hematological disorders and suggests granulysin as a possible diagnostic tool for CLH and CDLBCL-NOS of the face.
\end{abstract}

\section{Introduction}

Cutaneous lymphoid hyperplasia (CLH) is difficult to differentiate from primary malignant cutaneous lymphomas that may present as solitary nodules, and sometimes it requires much time to achieve a final diagnosis [1, 2]. However, early diagnosis of malignant lymphomas is important to ensure appropriate treatment and prevent the generalized

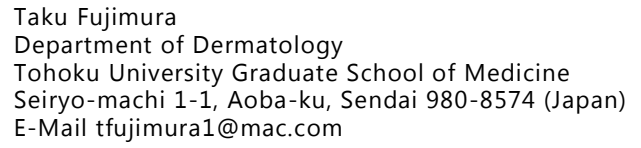


progress of the disease. Granulysin is a cationic molecule present in the granules of cytotoxic T lymphocytes and natural killer cells. Granulysin has homology with other cytotoxic molecules of the saponin-like protein family [3]. Indeed, several reports suggested that granulysin lyses various microbes and tumors in conjunction with perforin. In addition, a recent report also suggested that the expression of granulysin correlates with the prognosis of cancer patients, even in hematological disorders [4-6]. In this study, we examine the expression of cytotoxic molecules such as granulysin, TIA-1 and perforin in tumorinfiltrating lymphocytes (TILs) of CLH and cutaneous diffuse large B cell lymphoma, not otherwise specified (CDLBCL-NOS) of the face.

\section{Case Presentation}

During a 5-year period (2008-2012), 10 patients were diagnosed with CLH and 3 patients with CDLBCL-NOS at our department. We defined CLH and CDLBCL-NOS by their typical clinical courses, pathological features and the monoclonal rearrangement of immunoglobulin detected by Southern blot analysis of the skin biopsies. These samples were processed for single staining of CD8, CD161, granulysin, TIA-1 and perforin and developed with liquid permanent red (DAKO). The staining of infiltrated lymphocytes was examined in more than 5 random, representative fields of each section. The number of immunoreactive cells was counted using an ocular grid of $1 \mathrm{~cm}^{2}$ at a magnification of $\times 400$ by 2 dermatologists, who were blinded to the original histology and who reviewed all slides independently. Immunohistochemical staining revealed that TILs in CLH and CDLBCL-NOS are mainly composed of $\mathrm{CD}^{+}$cells (fig. 1a, b). Few CD161+ natural killer cells were detected in both CLH and CDLBCL-NOS (fig. 1c, d). Moreover, in CLH, the granulysin-bearing cells were densely infiltrated around the tumor (fig. 2a), while in CDLBCL-NOS, they were scattered around the tumor (fig. 2b). TIA-1+ $1^{+}$cells were detected (fig. 2c, d), but only few perforin ${ }^{+}$cells were present in either of the groups (data not shown). The number of granulysin ${ }^{+}$cells was significantly higher in CLH than in CDLBCL-NOS (103.5 \pm 11.9 vs. $20.7 \pm 3.2)$. There was no significant difference in the number of TIA- $1^{+}$and perforin ${ }^{+}$cells between CLH and CDLBCLNOS (TIA- $1: 187.7 \pm 43.8$ vs. $141.7 \pm 38.8$; perforin: $3.3 \pm 0.8$ vs. $7.5 \pm 2.4$ ) (fig. 3 ).

\section{Discussion}

The term cutaneous pseudolymphoma is used for a group of disorders with lymphocytic infiltration that histologically resembles cutaneous lymphoma [7]. Although these conditions were formally known as lymphadenitis benigna cutis, CLH is the currently recommended term $[7,8]$. Recently, Arai et al. [7] reported that the infiltrating cells in CLH are composed of a variety of mature lymphocytes, including CD3, CD4 and CD8. However, there has been no English report suggesting the presence of a subpopulation of CD8 in CLH. We previously reported a case of T-cell-/histiocyte-rich large B cell lymphoma, which is known as a subtype of diffuse large B cell lymphoma (DLBCL) with a better prognosis than other subtypes of DLBCL [9]. Interestingly, we also reported that TILs in T-cell-/histiocyte-rich LBCLs were mainly composed of $\mathrm{CD}^{+}$cells and abundant for granulysin-bearing cells. The expression of granulysin has been reported to correlate with the prognosis of cancer patients [4]. In hematological fields, Park et al. [5] reported that the pretreatment serum level of granulysin might have a prognostic value in patients with DLBCL. In addition, Kitamura et al. [4] reported the expression of granulysin in systemic anaplastic large cell lymphoma, which has 
Furudate et al:: Profiles of Cytotoxic T Lymphocytes in Cutaneous Lymphoid Hyperplasia of the Face

a relatively good prognosis, and concluded that the high expression of granulysin might correlate with the biological features of anaplastic large cell lymphoma. In the aggregate, these reports supported the hypothesis that the better prognosis of LBCL might be due to the tumor-infiltrating granulysin-bearing cytotoxic $\mathrm{T}$ cells.

To confirm this hypothesis, in this report, we employed immunohistochemical staining for TILs in CLH and CDLBCL-NOS of the face. As we expected, the number of granulysinbearing cells was significantly increased in CLH compared with CDLBCL-NOS. In contrast, for other functional markers of cytotoxic $\mathrm{T}$ cells such as TIA-1 and perforin, there was no significant difference between the two hematological disorders. Moreover, in contrast to the abundant $\mathrm{CD}^{+}$cells, only few $\mathrm{CD} 161^{+}$cells were detected in our cases. In summary, our present study might explain the different biological behaviors of these two hematological disorders and might suggest granulysin as a possible diagnostic tool for CLH and CDLBCLNOS of the face. However, further studies are needed to confirm this limited observation.

\section{Disclosure Statement}

The authors have no conflicts of interest to declare.

\section{References}

1 Holm N, Flaig MJ, Yazdi AS, Sander CA: The value of molecular analysis by PCR in the diagnosis of cutaneous lymphocytic infiltrates. J Cutan Pathol 2002;29:447-452.

-2 Fujimura T, Hidaka T, Hashimoto A, Aiba S: Dermoscopy findings of pseudolymphomatous folliculitis. Case Rep Dermatol 2012;4:154-157.

-3 Krensky AM, Clayberger C: Granulysin: a novel host defense molecule. Am J Transplant 2005;5:1789-1792.

-4 Kitamura N, Katagiri YU, Itagaki M, Miyagawa Y, Onda K, Okita H, Mori A, Fujimoto J, Kiyokawa N: The expression of granulysin in systemic anaplastic large cell lymphoma in childhood. Leuk Res 2009;33:908912.

-5 Park Y, Choi YJ, Park SJ, Lee SR, Sung HJ, Park KH, Kim SJ, Choi CW, Jung KY, Kim BS: Pretreatment serum level of 15-kDa granulysin might have a prognostic value in patients with diffuse large B cell lymphoma. Acta Haematol 2011;126:79-86.

-6 Pagès F, Berger A, Camus M, Sanchez-Cabo F, Costes A, Molidor R, Mlecnik B, Kirilovsky A, Nilsson M, Damotte D, Meatchi T, Bruneval P, Cugnenc PH, Trajanoski Z, Fridman WH, Galon J: Effector memory T cells, early metastasis, and survival in colorectal cancer. N Engl J Med 2005;353:2654-2666.

7 Arai E, Shimizu M, Hirose T: A review of 55 cases of cutaneous lymphoid hyperplasia: reassessment of the histopathologic findings leading to reclassification of 4 lesions as cutaneous marginal zone lymphoma and 19 as pseudolymphomatous folliculitis. Hum Pathol 2005;36:505-511.

-8 Baldassano MF, Bailey EM, Ferry JA, Harris NL, Duncan LM: Cutaneous lymphoid hyperplasia and cutaneous marginal zone lymphoma: comparison of morphologic and immunophenotypic features. Am J Surg Pathol 1999;23:88-96.

9 Kambayashi Y, Fujimura T, Tsukada A, Hashimoto A, Aiba S: Extranodal T-cell/histiocyte-rich B-cell lymphoma presenting primarily on the skin. Acta Derm Venereol 2012;92:637-639. 

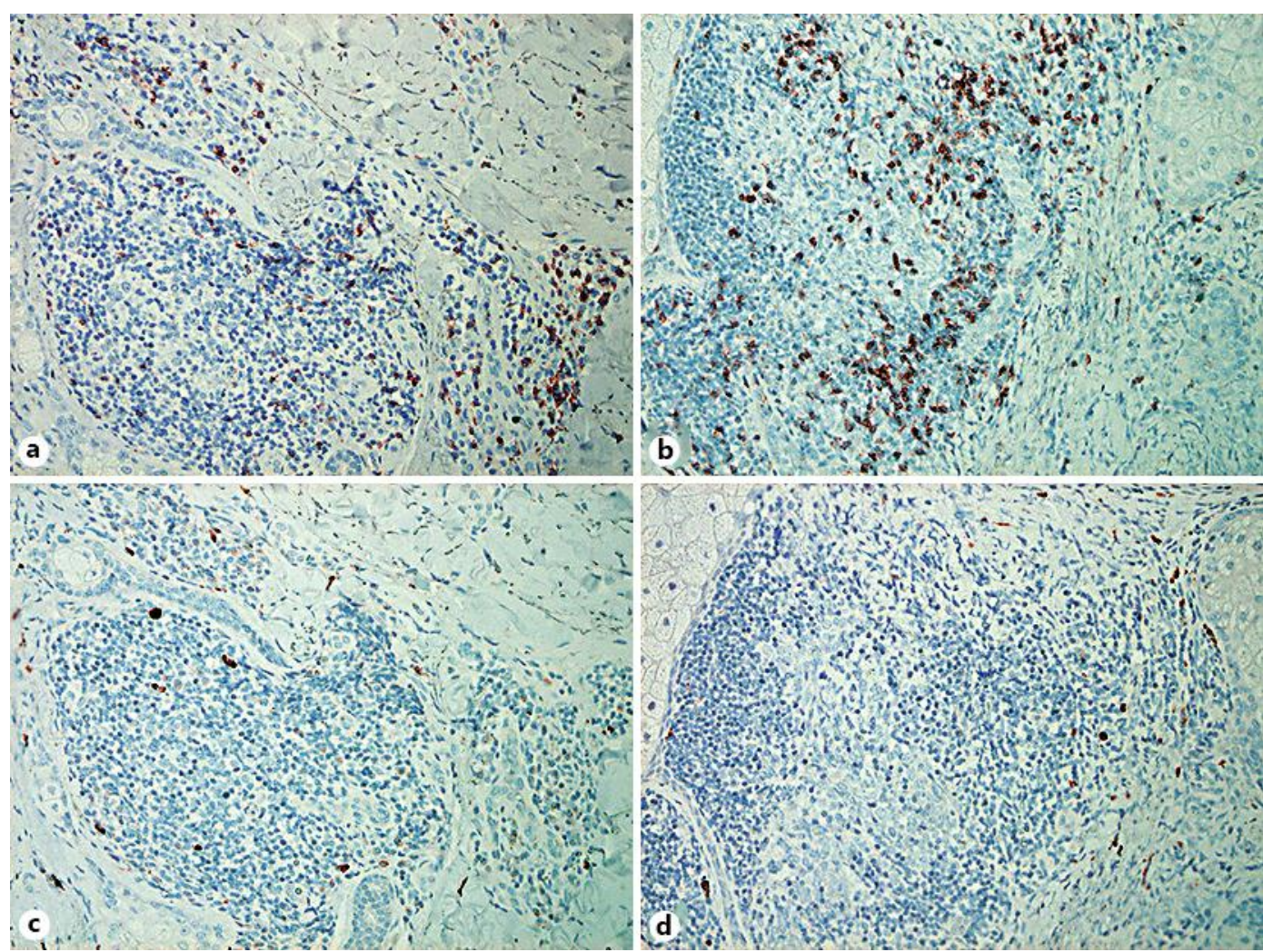

Fig. 1. CD8+ and CD161+ cells in CLH and CDLBCL-NOS of the face. Paraffin-embedded tissue samples from patients with $\operatorname{CLH}(\mathbf{a}, \mathbf{c})$ and CDLBCL-NOS $(\mathbf{b}, \mathbf{d})$ were deparaffinized and stained using an anti-CD8 antibody (a, b) or anti-CD161 antibody (c, d). a, b Original manifestation: $\times 200$. Sections were developed with liquid permanent red. 

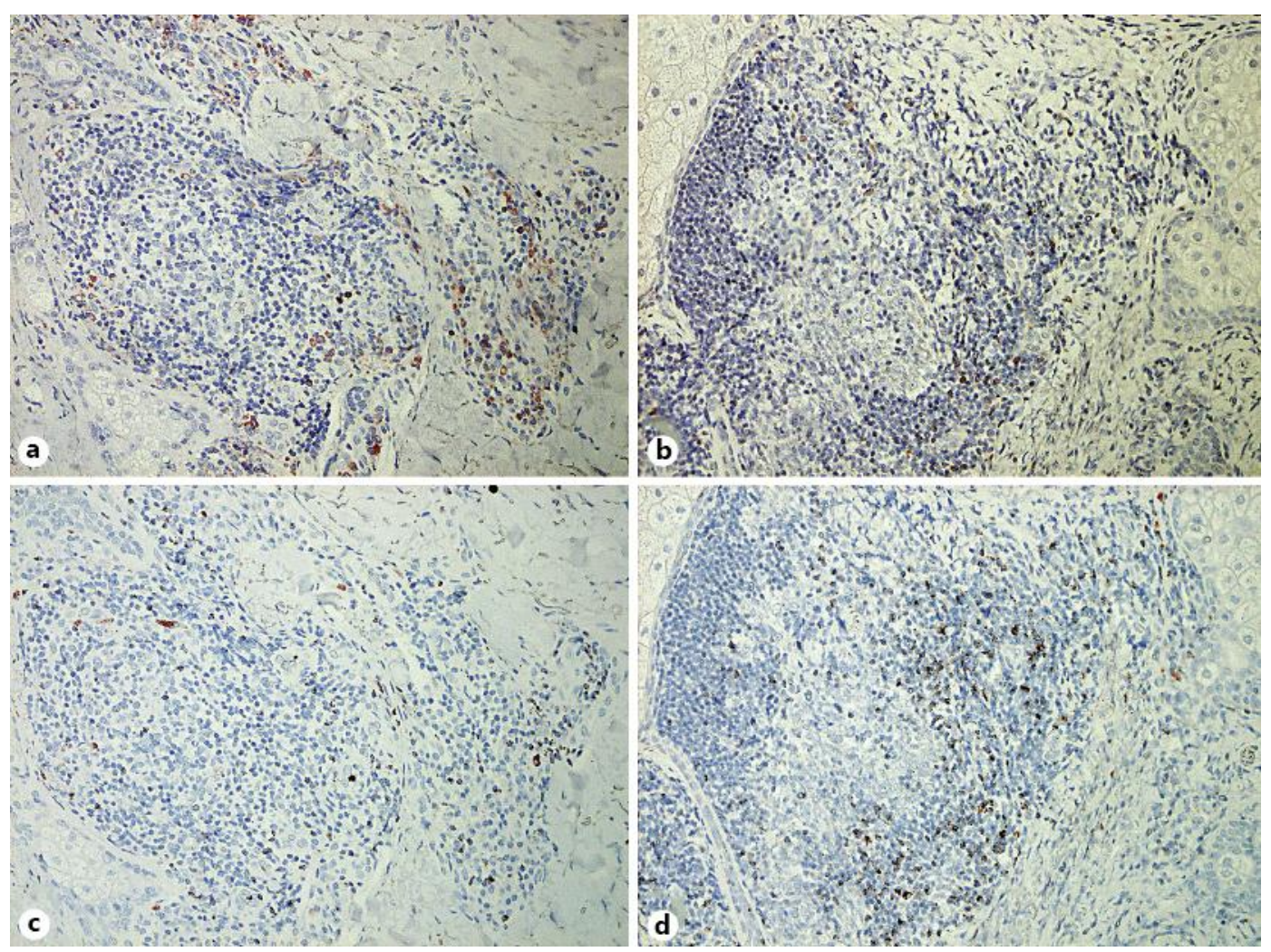

Fig. 2. Granulysin ${ }^{+}$and TIA-1+ cells in CLH and CDLBCL-NOS of the face. Paraffin-embedded tissue samples from patients with CLH (a, c) and CDLBCL-NOS (b, d) were deparaffinized and stained using an anti-granulysin antibody (a, b) or anti-TIA-1 antibody $(\mathbf{c}, \mathbf{d})$. a, b Original manifestation: $\times 200$. Sections were developed with liquid permanent red.

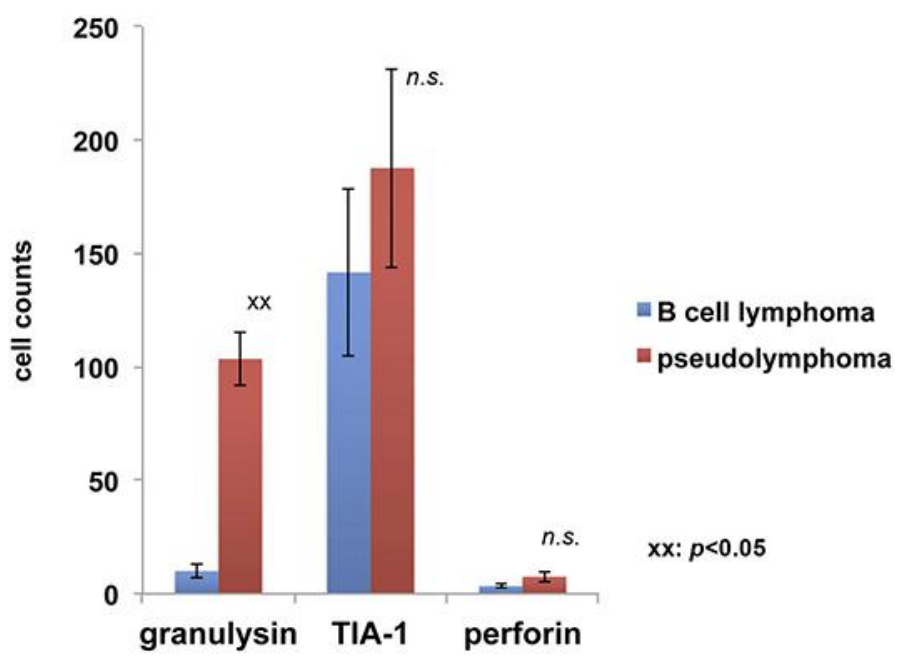

Fig. 3. Summary of the number of granulysin+, TIA-1+ and perforin ${ }^{+}$cells in CLH and CDLBCL-NOS of the face. Five representative fields of each section were selected from areas with dense lymphoid infiltration. The number of immunoreactive cells was counted using an ocular grid of $1 \mathrm{~cm}^{2}$ at a magnification of $\times 400$. Data are expressed as the means \pm SD of the cell number in each area. $x x p<0.05$; n.s. $=$ no significance. 